\title{
The microenvironment and resistance to personalized cancer therapy
}

Shigeo Masuda and Juan Carlos Izpisua Belmonte

We read with great interest the Perspectives article by Funda Meric-Bernstam and Gordon Mills (Overcoming implementation challenges of personalized cancer therapy. Nat. Rev. Clin. Oncol. 9, 542-548; 2012), ${ }^{1}$ in which the authors mainly focused on the intrinsic characteristics of the tumour for the design of personalized therapy for patients with cancer. However, three important papers recently published in Nature ${ }^{2,3}$ and Nature Medicine $e^{4}$ clearly demonstrate that, in addition to cell-autonomous factors (that is, intrinsic tumour characteristics), non-cell-autonomous determinants (that is, the tumour microenvironment) contribute to drug resistance in cancer therapy.

Firstly, hepatocyte growth factor (HGF) secretion from stromal cells was shown to be responsible for resistance in moleculartargeted cancer therapy. ${ }^{2,3}$ Straussman et al. ${ }^{2}$ found that, in BRAF-mutant melanoma, high levels of HGF in the stroma was associated with poor response to BRAF inhibition, suggesting tumour microenvironmentmediated drug resistance. Moreover, Wilson et al. ${ }^{3}$ reported that the plasma HGF concentration was inversely related to the response to BRAF inhibition in patients with $B R A F$-mutant melanoma. Interestingly, HGF is not simply a prognostic biomarker because the combined inhibition of BRAF and the HGF-cMET axis (for example, using HGF-neutralizing antibodies or the MET-inhibitory small molecule crizotinib) can reverse drug resistance in BRAF-mutant melanoma. ${ }^{2}$

Secondly, Sun et al. ${ }^{4}$ identified Wnt-16b protein as a chemoexposed stroma-derived factor that is essential for resistance to chemotherapy. In prostate cancer, chemotherapy was shown to cause DNA damage in stromal cells, and WNT16B expression was subsequently induced by NF- $\kappa \mathrm{B}$ after DNA damage. Secretion of Wnt-16b protein eventually reduced cancer-cell sensitivity to chemotherapy through non-cellautonomous determinants contributed by the tumour microenvironment.

Along this line, patient-derived tumour xenografts (PDTX), characterized by the maintenance of the original tumour architecture, have recently emerged as a model for cancer drug development. ${ }^{5}$ Interestingly, as human stromal cells in PDTX models can be replaced by murine stromal cells with the sequential passage from animal to animal, this opens the possibility to develop models to study the specificity and mechanisms of drug resistance between mouse and human stromal cells.

Taken together, the current data indicate that accurate and efficient predictions of response to cancer treatment will be incomplete unless a combined and integral approach towards the elucidation of tumoural-and microenvironmentalheterogeneity is undertaken. Such an approach must also involve a special focus on the secreted stromal factors responsible for drug resistance in each tumour subtype.

Gene Expression Laboratory, The Salk Institute for Biological Studies, 10010 North Torrey Pines Road, La Jolla, CA 92037, USA

(S. Masuda, J. C. Izpisua Belmonte). Center for Regenerative Medicine in Barcelona, Carrer del Doctor Aiguader 88, 08003 Barcelona, Spain (J. C. Izpisua Belmonte).

Correspondence to: S. Masuda

smasuda@salk.edu

\section{Competing interests}

The authors declare no competing interests.

1. Meric-Bernstam, F. \& Mills, G. B. Overcoming implementation challenges of personalized cancer therapy. Nat. Rev. Clin. Oncol. 9, 542-548 (2012).

2. Straussman, R. et al. Tumour micro-environment elicits innate resistance to RAF inhibitors through HGF secretion. Nature 487, 500-504 (2012).

3. Wilson, T. R. et al. Widespread potential for growth-factor-driven resistance to anticancer kinase inhibitors. Nature 487, 505-509 (2012).

4. Sun, Y. et al. Treatment-induced damage to the tumor microenvironment promotes prostate cancer therapy resistance through WNT16B. Nat. Med. 18, 1359-1368 (2012).

5. Tentler, J. J. et al. Patient-derived tumour xenografts as models for oncology drug development. Nat. Rev. Clin. Oncol. 9, 338-350 (2012). 